\title{
Le paysan et la mer. Ruralités littorales et maritimes en Europe au Moyen Âge et à l'Époque moderne
}

\section{Brice Rabot}

\author{
(2) OpenEdition \\ Journals \\ Édition électronique \\ URL : https://journals.openedition.org/abpo/6563 \\ DOI : $10.4000 / a b p o .6563$ \\ ISSN : 2108-6443 \\ Éditeur \\ Presses universitaires de Rennes
}

\section{Édition imprimée}

Date de publication : 10 décembre 2020

Pagination : 214-217

ISBN : 978-2-7535-8225-5

ISSN : 0399-0826

Référence électronique

Brice Rabot, «Le paysan et la mer. Ruralités littorales et maritimes en Europe au Moyen Âge et à l'Époque moderne », Annales de Bretagne et des Pays de l'Ouest [En ligne], 127-4 | 2020, mis en ligne le 10 décembre 2020, consulté le 06 janvier 2023. URL : http://journals.openedition.org/abpo/6563 ; DOI : https://doi.org/10.4000/abpo.6563 
de 250 à Paris. Pour devenir crêpier, des formations ont été mises en place par le Conseil régional mais, le plus souvent, c'est encore dans le cadre familial que la technique s'acquiert en observant les gestes d'une grand-mère ou d'une mère. À côté des crêperies installées dans chaque ville ou village, des entreprises se sont lancées dans la crêpe industrielle vendue en sachet dans les supermarchés et exportée parfois bien loin de la Bretagne. Enfin, il n'est quasiment pas de fêtes bretonnes sans crêpes : kermesses des écoles, pardons autour des chapelles, animations festives des associations pour récolter des fonds.

Abordant des thématiques variées et abondamment illustré, ce catalogue donne à comprendre l'essentiel sur la crêpe ou la galette, qu'elle soit de sarrasin ou de froment. Toutefois, les auteurs des 13 chapitres ont tendance à se répéter les uns les autres et un effort méritoire de synthèse aurait été le bienvenu.

Isabelle GUÉGAN

SARRAZIN, Jean-Luc, SAUZEAU, Thierry (dir.), Le paysan et la mer. Ruralités littorales et maritimes en Europe au Moyen Âge et à l'Époque moderne, Toulouse, Presses universitaires du Midi (Flaran, 39), 2019, 299 p.

Les $39^{\text {es }}$ journées internationales de Flaran avaient pour objet l'étude des paysanneries établies en rivage de mer pour interroger leurs spécificités. Campagnes littorales et espaces maritimes ne sont pas forcément liés, comme le soulignent avec force Jean-Luc Sarrazin et Thierry Sauzeau en introduction (p. 7-29). Sur quels critères se fonder pour l'affirmer? Les directeurs de publication commencent par rappeler quelques éléments essentiels : la part des navigants au sein d'une population permet de désigner l'identité maritime d'une campagne littorale, mais ne suffit pas à elle seule. La question des aménagements est tout aussi cruciale, renvoyant à l'examen des acteurs, de leurs logiques et de leurs modes d'adaptation pour exploiter au mieux les ressources. L'historien des ruralités littorales rejoint ici la géographie, qui s'est depuis longtemps intéressée au rapport des hommes à leur environnement. Jusque dans les années 1970-1980, peu d'études historiques ont été consacrées aux campagnes littorales/maritimes, si ce n'est dans le cadre de monographies se penchant sur le commerce ou l'économie des grandes villes portuaires. La recherche a connu de profondes inflexions au tournant des années 2000, dans le monde anglo-saxon en particulier (l'ouvrage de Peregrine Horden et Nicholas Purcell a mis en lumière le concept central de connectivity pour expliquer les connexions entre les différents sous-ensembles), invitant les autres chercheurs à poursuivre. Tel était l'objectif assigné au collectif de 13 chercheurs regroupés à Arthous les 13 et 14 octobre 2017 ( $39^{\text {es }}$ journées de Flaran).

La première partie s'ouvre sur la question des pêches et pêcheries avec l'intervention de Riccardo Rao, consacrée aux communaux marécageux et aux lagunes de l'Italie du Centre-nord au Moyen Âge (p. 33-50). L'auteur cherche à croiser le rôle des institutions publiques dans la gestion, en lien avec les exigences des marchés urbains, et le rôle de service public joué par les pêcheries en matière d'approvisionnement urbain. Partant du haut Moyen Âge, où les ecclésiastiques prédominent, les investigations conduisent $\mathrm{R}$. Rao aux XIV et $\mathrm{XV}^{\mathrm{e}}$ siècles, au moment où s'affirment les États princiers. La disparition de plusieurs pêcheries coïncide avec la désertion des espaces palustres et la reconversion vers l'élevage. Yannis Suire emboîte le pas du temps long pour retracer l'histoire des écluses et des autres équipements en Marais poitevin du Moyen Âge au XIX ${ }^{\mathrm{e}}$ siècle (p. 51-65). Les liens 
entre la côte et l'arrière-pays sont différents avec la question des opérations d'assèchement, conduites régulièrement aux $\mathrm{XII}^{\mathrm{e}}-\mathrm{XIII}^{\mathrm{e}}, \mathrm{XVII}^{\mathrm{e}}, \mathrm{XIX}^{\mathrm{e}}$ et dans la seconde moitié du $\mathrm{Xx}^{\mathrm{e}}$ siècle. Il en résulte des structures complexes, révélatrices des enjeux à différentes échelles : essentielles pour les habitants qui en tirent des bénéfices, les pêcheries le sont tout autant pour les seigneurs à travers les prélèvements opérés et les droits de propriété. Mais les pêcheries attirent les convoitises et les rivalités, ce qui précipite leur chute au XIXe siècle. "Symboles de la lutte perpétuelle des habitants du Marais avec les éléments qui les entourent " (p. 65), les pêcheries tombent en disgrâce. Il en est de même dans l'espace rétan, examiné par Jacques Bouchard, avec le cas tout à fait singulier des écluses à poissons (p. 67-86). Là encore éléments incontournables des paysages, les quelques vestiges conservés aujourd'hui (14 en exploitation sur un total de 140 recensées au XIX ${ }^{\mathrm{e}}$ siècle, p. 67) témoignent d'un savoir-faire et d'un lien étroit entre campagnes littorales et espaces maritimes. Prolongement en mer du territoire terrestre, approprié et mis en valeur par les communautés rurales, les écluses à poissons interrogent les pratiques et les liens entre les hommes. Elles sont peu à peu tombées dans l'oubli, mais les pouvoirs publics semblent prendre conscience, depuis 2011 pour l'île de Ré, de la nécessité de les protéger. Daniel Faget clôt cette première partie avec le récit du pillage des fontaines de Salses (Roussillon) en 1758 (p. 87-102). Cette affaire éclaire trois éléments distincts et inter-reliés en même temps : la diversité des régimes de pêche dans les milieux lagunaires sous l'Ancien Régime, la résistance d'une communauté rurale face à l'intervention extérieure (qu'elle voit comme une confiscation injustifiée) et les liens avec les paysanneries du Roussillon (échelle plus large). Cet épisode, qui ne débouche pas sur un procès avec le règlement infrajudiciaire par les notables, souligne le poids des pratiques de pêche dans la culture villageoise dans le contexte particulier du XviII ${ }^{\mathrm{e}}$ siècle, où les droits font l'objet de nombreuses convoitises.

La deuxième partie est centrée sur l'exploitation des terres avec les questions des agricultures littorales. Tim Soens ouvre les débats en se penchant sur la pluriactivité des sociétés littorales du $\mathrm{Xl}^{\mathrm{e}}$ au Xvile siècle autour de la mer du Nord (p. 105-125). Il s'agit de dépasser la vision traditionnelle d'espaces souvent associés à la pauvreté : la mer du Nord compte les régions maritimes pluriactives parmi les plus riches d'Europe aux $\mathrm{XIII}^{\mathrm{e}}-\mathrm{XV}^{\mathrm{e}}$ siècles. Comment l'expliquer? Les petites exploitations familiales et la liberté personnelle sont fondamentales, au même titre que l'intégration à des réseaux commerciaux à différentes échelles. De profonds changements interviennent vers 1450 avec la spécialisation accrue, la division de la société agraire (l'auteur parle à ce sujet de " capitalisme agraire ") et, surtout, la division entre la terre et la mer. Jean-Claude Hocquet et Inês Amorim se penchent eux sur le cas très particulier des salines. Onservant les rivages adriatiques, JeanClaude Hocquet met en exergue les liens entre sel, société et habitat des sauniers (p. 127-147). Ses propos restent cependant limités à un espace précis (l'Adriatique), afin de garder une cohérence et pour scruter plus précisément les liens avec la société urbaine. L'étude des statuts urbains, conjuguée aux données issues des campagnes et du négoce, permet de retracer une diversité de situations où les interactions entre villes, campagnes et salines sont majeures. Inês Amorim revient sur les comportements, en écho à la contribution précédente, pour mieux souligner les singularités de l'espace portugais (p. 149-172). L'adaptation au marché s'avère très complexe : la concurrence exacerbée entre les centres traditionnels de production européens dans les années 1760-1769 conduit à adopter de nouveaux dispositifs réglementaires (protectionnistes) pour continuer d'attirer les négociants du nord de l'Europe, qui se tournent vers d'autres sources que le Portugal. Gérard Le Bouëdec questionne l'agriculture littorale française du XVII ${ }^{\mathrm{e}}$ au $\mathrm{XIX}^{\mathrm{e}}$ siècle (p. 173-203). 
L'idée est de combler un vide historiographique en incitant les ruralistes à tourner leurs regards vers les aménagements mis en œuvre de l'estran vers l'arrière-pays. De nombreux exemples viennent fort à propos appuyer la démonstration, qui se tourne peu à peu vers la pluriactivité paysanne. Gérard Le Bouëdec insiste sur la nécessité de partir de l'océan pour élargir les focales.

La troisième partie est consacrée à l'océan en partant de l'angle des risques. Sébastien Perisse évoque les vimaires en Saintonge aux XIV ${ }^{\mathrm{e}} \mathrm{XVI}^{\mathrm{e}}$ siècles (p. 207225). Il les rapproche des catastrophes qui, par leur soudaineté, leur violence et leurs destructions, ébranlent les sociétés littorales. Très bien documentée, cette présentation fait la part belle aux adaptations et au pragmatisme des sociétés. La notion de résilience (p. 222), empruntée aux autres sciences humaines, est malgré tout peu explorée, ce qui aurait élargi les perspectives. Emmanuelle Charpentier complète ceci avec les "sables volages " du Léon entre les XVII ${ }^{\mathrm{e}}$ et XIX ${ }^{\mathrm{e}}$ siècles (p. 227-246). Là encore, l'objectif est de mettre en évidence le poids des stratégies individuelles et collectives pour lutter contre un phénomène insidieux et particulièrement pénalisant pour le Léon. Les communautés locales font appel au XVIII ${ }^{\mathrm{e}}$ siècle aux experts extérieurs des Ponts-et-Chaussées pour gagner cette lutte, avant que la période révolutionnaire ne vienne y mettre un brutal coup d'arrêt. Les anciennes pratiques (pacage notamment) et l'absence d'entretien fragilisent les initiatives, qui en restent au stade d'expérimentations avant le $\mathrm{XIX}^{\mathrm{e}}$ siècle. Pierre Caillosse parvient à d'autres conclusions pour ce qui concerne les marais de Soulac aux XVIII -XIX ${ }^{\mathrm{e}}$ siècles (p. 247-262). Espaces particulièrement vulnérables aux assauts marins, mais à fort potentiel, les marais de Soulac connaissent bien des vicissitudes. L'outil informatique offre ici des vues particulièrement poignantes en croisant les éléments tirés de cartes anciennes avec ceux du terrain. L'on suit, pas à pas, ces évolutions, enclenchées dès le début du XVIII siècle, qui conduisent à un recul lent mais inexorable de la saliculture au profit de la céréaliculture, beaucoup plus lucrative du fait des droits fonciers.

La quatrième et dernière partie s'attache au cabotage. Gilbert Buti pose la question de " la mer au service de la terre en Méditerranée nord-occidentale (XVII XIX ${ }^{e}$ siècles) " (p. 265-281). De nombreux exemples viennent étayer ses propos pour démontrer à quel point les campagnes littorales et la mer ont entretenu des liens ambivalents, bien plus complexes que les sources ne le laisseraient penser de prime abord. Les terroirs littoraux s'étendent bien au-delà des côtes avec les trafics de divers ordres, rejoignant la notion d'interface (que l'auteur n'emploie toutefois pas). Werner Scheltjens ferme ce recueil avec le réexamen des transports maritimes en Hollande et dans le nord de l'Allemagne avec les Comptes du Sund (p. 283-293). L'interaction avec le commerce maritime international, qui ne cesse de se développer après 1400, conduit à des bouleversements sans précédents avec le passage à une seule occupation dominante toute l'année, accompagnée d'une spécialisation dans un secteur dominant. Les différents estuaires des Pays-Bas sont de plus en plus intégrés au long du XvIII ${ }^{e}$ siècle, en lien avec le développement des services de transport maritime.

$\mathrm{Au}$ total, cet ouvrage ouvre de stimulantes perspectives pour les chercheurs ruralistes, les invitant à dépasser le seul cadre terrien pour analyser les réseaux, les stratégies en lien avec l'espace maritime, en partant pour cela de cas précis. Toutes les campagnes littorales ne tournent malgré tout pas leurs regards vers la mer et l'océan, ce qui explique le foisonnement des études de cas, dans la droite ligne des journées internationales d'histoire rurale de Flaran. L'on peut toutefois regretter la timidité de certains à employer des concepts bien ancrés et régulièrement mobilisés par d'autres sciences humaines (nous pensons ici en particulier à la géographie), comme Jean-Luc Sarrazin et Thierry Sauzeau invitaient à le faire en 
introduction. Cette dernière remarque n'enlève rien à la qualité de l'ouvrage, qui saura intéresser bien au-delà des seuls cercles ruralistes, avec des présentations méthodologiques rigoureuses et utiles pour les historiens.

Brice RABOT

Guillot DE Suduiraut, Sophie (dir.), Le retable anversois de la cathédrale de Rennes. Un chef-d'œuvre révélé, Rennes, Presses universitaires de Rennes, 2019, 207 p.

Quelque peu incongru dans un grand vaisseau néo-classique paré d'ors et de stucs au temps de $M^{\text {gr }}$ Brossays Saint-Marc, le grand retable gothique de la cathédrale Saint-Pierre fait aujourd'hui l'objet d'une véritable redécouverte dont témoigne ce très bel ouvrage des Presses universitaires de Rennes. L'œuvre était jusqu'ici mal servie par sa disposition dans une chapelle latérale qui ne lui donnait ni lumière, ni recul; le retable se trouvait confiné derrière un écran vitré dont l'objectif de sécurité n'avait pas empêché le vol de plusieurs pièces en 2007. La restauration de l'ensemble des décors de la cathédrale, remarquablement menée depuis 2009 par l'État maître d'ouvrage, a été l'occasion de concrétiser le projet ancien de faire de cet objet exceptionnel le joyau d'un Trésor en libre accès dans l'ancienne sacristie Nord.

Pour la première fois depuis 1872 , l'occasion était ainsi donnée de déposer le retable, de le soumettre à des analyses approfondies et de le restaurer selon les principes actuellement reconnus. À la joie d'admirer cette œuvre comme jamais, le livre ajoute donc le plaisir d'accéder à tout ce que le chantier a révélé : les textes, les clichés photographiques et la séduisante maquette sont en tous points remarquables. L'ouvrage, dirigé par Sophie Guillot de Suduiraut, ancienne conservatrice en chef au département des Sculptures du musée du Louvre, a bénéficié de sa compétence en matière de retables qu'elle dit " brabançons ", qualificatif mieux accordé à la réalité politique du début du Xvi ${ }^{\mathrm{e}}$ siècle que le trop extensif " flamand ». Le livre réunit également les signatures des conservateurs des Monuments Historiques en Bretagne (Henry Masson, Christine Jablonski, Cécile Oulhen) et l'expertise technique des maîtres d'œuvre, français et belges, des études et de la restauration proprement dite.

L'origine anversoise du retable, avancée depuis les années 1950, se trouve ainsi confirmée et précisée par le repérage sur la boiserie de pas moins de 34 mains et 2 châteaux (armes d'Anvers) qui sont autant de " marques de garantie " apposées au fer. Tous les indices concordent pour dater le retable des environs de 1520. D'une part, l'analyse dendrochronologique a révélé l'origine du bois - un excellent chêne dit waghenschot, provenant de l'est de la Baltique - et en a établi l'abattage entre 1512 et 1522 . D'autre part, l'analyse proprement stylistique situe l'œuvre rennaise par rapport aux 180 retables anversois du Xvi ${ }^{\mathrm{e}}$ siècle conservés aujourd'hui du Portugal à la Suède (dont une vingtaine en France). Le retable de Rennes correspond à un certain apogée des ateliers anversois, où se conjuguent qualité des matériaux, production intensive, maîtrise du style. Celui-ci peut se définir par une élégance gracieuse, un peu affectée parfois, mais sans le maniérisme sensible à partir de 1530; sans vocabulaire italianisant encore, sinon dans un ou deux hypothétiques détails. On savait déjà, en particulier depuis la thèse de Gildas Durand, combien la Bretagne avait été réceptive à la production artistique des anciens Pays-Bas, avec lesquels la péninsule entretenait d'intenses relations commerciales. Christine Jablonski actualise ici, de façon précise, la liste des sculptures bretonnes d'impor- 